Pacific Journal of Mathematic 


\section{THE PRODUCT FORMULA FOR SEMIGROUPS DEFINED BY FRIEDRICHS EXTENSIONS}

\section{WILLIAM G. FARIS}

Suppose that $A$ and $B$ are linear operators which generate semigroups on a Hilbert space. Then $A+B$ may be far from being a generator. Nevertheless, a generator may sometimes be defined by adding operators corresponding to $A$ and $B$, but with values in a larger Hilbert space, and then restricting the sum to the original Hilbert space. Here an explicit product formula in terms of the semigroups generated by $A$ and $B$ is shown to converge to a semigroup, which is that given by this sum. This result has application to the perturbation theory of partial differential equations. This is illustrated by the Feynman path integral representation of the solution of the Schrödinger equation with potential term containing singularities.

Let $A$ and $B$ be linear operators generating semigroups $\exp (t A)$ and $\exp (t B)$ in a Banach space. Then under suitable conditions

$$
\exp (t(A+B))=\lim _{n \rightarrow \infty}\left(\exp \left(\frac{t}{n} A\right) \exp \left(\frac{t}{n} B\right)\right)^{n} .
$$

This is the Trotter [28] product formula. Though it is a theorem in perturbation theory, it is also related to the Feynman path integral representation of solutions of partial differential equations and provides the best mathematical realization of this idea presently known.

Feynman [10] considered the Schrödinger equation of quantum mechanics

$$
i \frac{d u(t)}{d t}=-\frac{1}{2 m} \Delta u(t)+V u(t)
$$

for $u(t)$ in $L^{2}\left(\boldsymbol{R}^{3}\right)$ for each $t$ and with initial condition $u(0)=u$. Here $\Delta$ is the Laplacian and $V$ is a real valued function on $\boldsymbol{R}^{3}$ multiplying $u(t)$. If we take $A=(i / 2 m) \Delta$ and $B=-i V$, the Trotter formula may be applicable. We have explicitly

$$
\begin{aligned}
& \exp \left(\frac{i t}{2 m} \Delta\right) u(x) \\
& =(2 \pi i t / m)^{-3 / 2} \int_{R^{3}} \exp \left[i \frac{1}{2} m\left(|x-y|^{2} / t\right)\right] u(y) d y .
\end{aligned}
$$

Thus, as Nelson [24] observed, the formula representing the solution is 


$$
\begin{aligned}
u(x, t)= & \exp \left(\frac{i t}{2 m} \Delta-i t V\right) u(x) \\
= & \lim _{n \rightarrow \infty}\left(\exp \left(\frac{i t}{2 m n} \Delta\right) \exp \left(-\frac{i t}{n} V\right)\right)^{n} u(x) \\
= & \lim _{n \rightarrow \infty} \int \cdots \int \exp \left[i \sum_{j=1}^{n}\left\{\frac{1}{2} m \frac{\left|x_{j}-x_{j-1}\right|^{2}}{(t / n)^{2}}-V\left(x_{j-1}\right)\right\} \frac{t}{n}\right] \\
& \times u\left(x_{0}\right)(2 \pi i t / n m)^{-(3 n / 2)} d x_{0} \cdots d x_{n-1},
\end{aligned}
$$

where $x_{n}=x$.

This is formally an integral over the space of all paths $x(\tau)$, $0 \leqq \tau \leqq t$, in $\boldsymbol{R}^{3}$ such that $x(t)=x$ of

$$
\exp \left[i \int_{0}^{t}\left\{\frac{1}{2} m\left(\frac{d x(\tau)}{d \tau}\right)^{2}-V(x(\tau))\right\} d \tau\right] u(x(0))
$$

which was Feynman's solution.

Various other attempts to provide a definition of Feynman integral have been made by Daletzski [6], who uses the Trotter formula, and Cameron [4] and Ito [14], who both apply integral transforms. Feldman [9] and Babbit [1] study the Schrodinger equation by analytic continuation in the time parameter from a Wiener integral solution of a parabolic equation, and Nelson [24] uses analytic continuation in the mass parameter. Expository treatments of the Feynman integral may also be found in Kac [15], Gelfand and Yaglom [12], and McShane [22].

Here we prove a version of the Trotter formula for semigroups, by Hilbert space methods, avoiding the use of Wiener integrals. We consider a certain class of perturbations $B$ of $A$ so singular that the sum $A+B$ need not be densely defined when $A$ and $B$ are regarded as (partially defined) operators acting in the original Hilbert space. However, the quadratic form of the perturbing operator $B$ is assumed smaller than the quadratic form of $A$, so that the sum may be defined using the method of the Friedrichs extension. (See Lions [19] and Nelson [25].) The Trotter formula is proved for parabolic equations and continuity in the mass parameter gives a definition of Feynman integral for the Schrödinger equation. Thus we get an explicit perturbation formula under conditions much less restrictive than are needed for the usual series formulas.

Definition by continuation in a parameter is at least in keeping with tradition, having been frequently used since Cameron adopted it in the first mathematical paper on Feynman integrals. The analytic continuation technique used by Nelson [24] allows the perturbing term $V$ to be more singular than considered here. However, in the present context there is no possibility of a set of exceptional masses for which the solution may fail. 
The range of applicability of the abstract theorems is illustrated by the Schrödinger equation in $n$ dimensions.

1. The Friedrichs extension method. In studying differential equations, Lions [19] and Nelson [25] have exploited the special properties of semi-bounded operators on Hilbert space first noticed by Friedrichs. Some of these constructions are sketched in the following. The idea is to use the operators to construct abstract analogs of Sobolev spaces, and to consider continuous operators from these spaces to their duals. The use of operators from a space to its dual instead of the quadratic forms on the space that Friedrichs used gives a mild formal simplification.

Notation. If $X$ and $Y$ are Banach spaces, $L(X, Y)$ is the space of all continuous linear transformations from $X$ to $Y$. $L(X)=L(X, X)$. In general, an operator $A$ from $X$ to $Y$ will be a linear transformation from some vector subspace $D(A)$ of $X$ into $Y$. Thus $A$ is in $L(X, Y)$ if and only if $D(A)=X$ and $A$ is continuous. We refer to an operator $A$ from $X$ to $X$ as an operator in $X$.

If $K$ is a Hilbert space, by the conjugate dual space $K^{*}$ we will mean the Hilbert space of continuous anti-linear functionals on $K$. We write $(v, u)$ for the value of $v$ in $K^{*}$ on $u$ in $K$.

Definition. A sector $S$ of the complex plane is a closed convex subset bounded by two rays meeting at 0 . Let $A$ be an operator from $K$ to $K^{*} . A$ is said to be of type $S$ if $(A u, u) \in S$ for all $u$ in $D(A)$. $A$ is dissipative if it is of type left half-plane.

Let $H$ be a Hilbert space with a given inner product. Use it to identify $H^{*}$ with $H$. Let $A$ be an operator in $H$. The Hille-Yosida theorem implies that $A$ is the infinitesimal generator of a (strongly continuous) contraction semigroup if and only if

(i) $D(A)$ is dense in $H$,

(ii) $A$ is dissipative, and

(iii) There is a real $\lambda_{0}$ such that $(\lambda-A)^{-1}$ is in $L(H)$ for all $\lambda>\lambda_{0}$.

This semi-group will be written $\exp (t A), t \geqq 0$. (Basic references for semigroup theory are Dunford and Schwartz [8], Nelson [25], and Yosida [29]. For this version of the Hille-Yosida theorem see Nelson [24] or Lumer and Phillips [21].)

Definition. Let $H$ be a Hilbert space with given inner product. Use this inner product to identify $H$ with its (conjugate) dual space. Let $H^{1}$ be another Hilbert space with $H^{1} \subset H$ as a dense linear subspace such that the injection is continuous. (No distinguished norm 
in the family of equivalent norms on $H^{1}$ is specified.) Let $H^{-1}$ be the (conjugate) dual space of $H^{1}$. The dual of the given injection gives a continuous injection of $H$ into $H^{-1}$ which we may use to identify $H$ as a dense linear subspace of $H^{-1}$. The triple $H^{1} \subset H \subset H^{-1}$ constructed in this way will be called a scale of Hilbert spaces.

Notation. Let $(v, u)$ be the given inner product on $H$. Then $v$ in $H$ is identified (by the Riesz theorem) with the functional in $H^{*}$ whose value on $u$ in $H$ is given by $(v, u)$. Now such a functional in $H^{*}$ is identified with the functional in $H^{-1}$ whose value on $u$ in $H^{1} \subset H$ is given by the same expression. Thus if $v$ is any element of $H^{-1}$ and $u$ is in $H^{1}$ we may consistently write $(v, u)$ or $(\overline{u, v})$ for the value of $v$ on $u$.

Proposition 1. Let $A$ be a densely defined operator in $H$ of type $S$, where $S-\{0\}$ is contained in the open left halfplane. Then there is a unique scale $H^{1} \subset H \subset H^{-1}$ such that

(1) $D(A)$ is dense in $H^{1}$.

(2) $A$ maps $D(A) \subset H^{1}$ continuously into $H^{-1}$.

Thus $A$ extends by continuity to an operator $\hat{A}$ in $L\left(H^{1}, H^{-1}\right)$. Let $J=\operatorname{Re}-\hat{A}+\lambda$ for any $\lambda>0$.

(3) The inner product $(J u, v)$ defines an equivalent norm on $H^{1}$.

Proof. Let $B=-A+\lambda$. Give $D(A)$ the norm $\|u\|_{i}^{2}=\operatorname{Re}(B u, u)$. Since $A$ is of type $S$, there is a constant $c>0$ with $|\operatorname{Im}(A u, u)| \leqq$ $c \operatorname{Re}(-A u, u)$. Thus $|(A u, u)| \leqq\left(1+c^{2}\right)^{1 / 2} \operatorname{Re}(-A u, u)$. Since $\operatorname{Re}(-A u, u) \leqq(u, u)_{1}$, it follows that there is an $M>0$ such that $|(A u, v)| \leqq M\|u\|_{1}\|v\|_{1}$ (Lang [18, p. 114]).

Now let $H^{1}$ be the completion of $D(A)$ in this norm. The identity mapping of $D(A)$ into $H$ extends by continuity to a mapping $\tau$ in $L\left(H^{1}, H\right)$. It remains to prove that $\tau$ is an injection.

Let $w$ be in $H^{1}$ with $\tau(w)=0$. Let $\varepsilon>0$. Choose a neighborhood $N$ of $w$ such that for $u, v$ in $N \cap D(B), \operatorname{Re}(B u, u)$ is within $\varepsilon / 2$ of $\operatorname{Re}(B v, u)$. Fix $v$ in $N \cap D(B)$ and let $u \rightarrow w$ in $H^{1}$. Then $u=$ $\tau(u) \rightarrow \tau(w)=0$ in $H$. So for $u$ near $w, \operatorname{Re}(B v, u)$ is within $\varepsilon / 2$ of 0 . Thus $\|u\|_{1}^{2}=\operatorname{Re}(B u, u)$ is within $\varepsilon$ of 0 . This shows that $\|w\|_{1}^{2} \leqq \varepsilon$. Since $\varepsilon$ was arbitrary, $w=0$.

Now since $\hat{A} \in L\left(H^{1}, H^{-1}\right), \hat{A}^{*} \in L\left(H^{-1 *}, H^{1 *}\right)=L\left(H^{1}, H^{-1}\right)$. Thus $\operatorname{Re} \hat{A}=(1 / 2)\left(\hat{A}+\hat{A}^{*}\right) \in L\left(H^{1}, H^{-1}\right)$ and is self-adjoint. If we let $J=$ $-\operatorname{Re} \hat{A}+\lambda$, the inner product on $H^{1}$ is given by $(u, v)_{1}=(J u, v)$.

Proposition 2. Let $H^{1} \subset H \subset H^{-1}$ be a scale. Let $B$ be in $L\left(H^{1}, H^{-1}\right)$ and suppose that for some $c>0,|(B u, u)| \geqq c\|u\|_{1}^{2}$ for all $u$ in $H^{1}$. Then $B$ is an isomorphism of $H^{1}$ onto $H^{-1}$. 
Proof. $R(B)$ is dense. Otherwise there is a $w$ in $H^{1}$ such that $(B u, w)=0$ for all $u$ in $H^{1}$. In particular $(B w, w)=0$. Hence $\|w\|_{1}=0, w=0$.

$R(B)$ is closed. For $\|B u\|_{-1}\left|\|u\|_{1} \geqq\right|(B u, u) \mid \geqq c\|u\|_{1}^{2}$, so $B$ is one-to-one with bounded inverse. Thus $R(B)$ is isomorphic to $H^{1}$ and hence is complete.

Proposition 3. Let $H^{1} \subset H \subset H^{-1}$ be a scale. Let $A \in L\left(H^{1}, H^{-1}\right)$. Let $D\left(A_{0}\right)=\left\{u \in H^{1}: A u \in H\right\}$ and $A_{0}$ be the restriction of $A$ to $D\left(A_{0}\right)$. Suppose that $A$ is dissipative and satisfies for some $\lambda>0$ and $c>0$

$$
\mid(-A+\lambda) u, u)|\geqq c| \mid u \|_{1}^{2} .
$$

Then $A_{0}$ is the infinitesimal generator of a contraction semigroup.

Proof. $-A+\lambda$ is an isomorphism from $H^{1}$ onto $H^{-1}$. Since $H$ is dense in $H^{-1},(-A+\lambda)^{-1} H$ is dense in $(-A+\lambda)^{-1} H^{-1}$. This says that $D\left(A_{0}\right)$ is dense in $H^{1}$ and hence in $H$.

It also follows that $-A_{0}+\lambda$ is onto $H$. The Hille-Yosida theorem implies the conclusion.

2. The product formula for abstract parabolic equations. In general the semigroup $\exp t A$ generated by an operator $A$ gives a solution to the initial value problem for the differential equation $(d u(t) / d t)=A u(t)$. A typical case is when $A$ is the Laplacian or a similar elliptic differential operator. Then the differential equation is parabolic and the solution $u(t)=\exp (t A) u$ tends to be smooth for $t>0$. Such characteristic properties of parabolic equations are due to the fact that spectrum of $A$ is in a sector bounded by rays in the open left half-plane and are found in the abstract case when this type of condition on the operator is satisfied. Here the product formula is proved for perturbations of such abstract parabolic equations where the perturbed sum is defined by the Friedrichs extension method.

Recall that $B$ is a densely defined maximal dissipative operator in $H$ if and only if $B$ is the infinitesimal generator of a contraction semigroup. If $B$ is such an operator, then $-B$ has a square root (Kato [16], Balakrishnan [3]). However, note that $(-B)^{1 / 2}$ and $\left(-B^{*}\right)^{1 / 2}=(-B)^{1 / 2^{*}}$ need not have the same domain of definition (Lions [20]).

THeORem 1. Let $H$ be a Hilbert space. Let $A$ be the infinitesimal generator of a contraction semigroup in $H$. Assume that $A$ is of type $S$, where $S-\{0\}$ is contained in the open left half-plane. Let $H^{1} \subset H \subset H^{-1}$ be the scale associated with $A$. A extends by continuity to an operator $\hat{A}$ in $L\left(H^{1}, H^{-1}\right)$. 
Let $B$ be the infinitesimal generator of a contraction semigroup in $H$. Assume that

(1) $D(B) \cap H^{1}$ is dense in $H^{1}$ and $B$ on this domain extends by continuity to an operator $\hat{B}$ in $L\left(H^{1}, H^{-1}\right)$.

(2) $(-B)^{1 / 2}$ and $\left(-B^{*}\right)^{1 / 2}$ may each be restricted to some domain dense in $H^{1}$ on which they are continuous from $H^{1}$ to $H$.

Then $\hat{A}+\hat{B}$ has a restriction $C$ which is the infinitesimal generator of a contraction semigroup in $H$. As $n \rightarrow \infty$ the products

$$
(\exp (t / n A) \exp (t / n B))^{n}
$$

converge to $\exp t C$ in the strong operator topology of $H$.

Note. In case $B$ is a normal operator in $H$ (as it is in most applications), the condition (3) below implies (1) and (2).

(3) $D(|B|) \cap H^{1}$ is dense in $H^{1}$ and $|B|$ on this domain extends by continuity to an operator in $L\left(H^{1}, H^{-1}\right)$.

Proof. (1) By the spectral theorem, for $u$ in $D(B)=D(|B|)$, we have $|(B u, u)| \leqq(|B| u, u)$. Since the quadratic form of $|B|$ is bounded on $H^{1}$, then so is the one for $B$.

(2) For $u$ in $D(|B|)$ we have $\left\|(-B)^{1 / 2} u\right\|^{2}=\left\|\left(-B^{*}\right)^{1 / 2} u\right\|^{2}=$ $(|B| u, u)$.

Proof of Theorem 1. Consider $\hat{C}=\hat{A}+\hat{B} \in L\left(H^{1}, H^{-1}\right)$. Since $\hat{A}$ is of type $S$ and $B$ is dissipative, $\hat{C}$ is dissipative. In addition we have

$$
\operatorname{Re}((-\hat{C}+\lambda) u, u) \geqq \operatorname{Re}((-\hat{A}+\lambda) u, u) \geqq c\|u\|_{1}^{2} .
$$

Thus $\hat{C}$ has a restriction to an operator $C$ which is the infinitesimal generator of a contraction semigroup $\exp t C$ in $H$. Note for future use that it follows from the above inequality that $\operatorname{Re}((-\hat{C}+\lambda) u, u)$ defines an equivalent norm on $H^{1}$.

Since $\hat{A}$ is of type $S$, there is a $c>0$ such that $|\operatorname{Im}(\hat{A} u, u)| \leqq$ $c \operatorname{Re}(-\hat{A} u, u)$. Thus

$$
\begin{aligned}
|\operatorname{Im}(\hat{C} u, u)| & \leqq|\operatorname{Im}(\hat{A} u, u)|+|(\hat{B} u, u)| \\
& \leqq c \operatorname{Re}(-\hat{A} u, u)+b \operatorname{Re}((-\hat{A}+1) u, u) \\
& \leqq c \operatorname{Re}(-\hat{C} u, u)+b \operatorname{Re}((-\hat{C}+1) u, u) .
\end{aligned}
$$

It follows that for some $a>0$,

$$
\mid \operatorname{Im}((\widehat{C}-1) u, u) \leqq a \operatorname{Re}(-(\widehat{C}-1) u, u) .
$$

Thus $\hat{C}-1$ is of type $S^{\prime}$ with $S^{\prime}-\{0\}$ contained in the open left half-plane. 
Introduce the notation $P^{t}=\exp t A, Q^{t}=\exp t B$, and $R^{t}=\exp t C$. $A$ and $C-1$ both have restrictions on their type which imply that $P^{t}$ and $R^{t}$ are holomorphic semigroups for $t>0$.

We prove the equivalent statement that $\lim _{n \rightarrow \infty}\left(Q^{t / n} P^{t / n}\right)^{n}=R^{t}$.

Let $h=t / n, T^{h}=Q^{h} P^{h}, \mathrm{~S}_{n}=\left(T^{h}\right)^{n}-\left(R^{h}\right)^{n}$. We want to show that $S_{n} \rightarrow 0$ strongly as $n \rightarrow \infty$.

Write

$$
\begin{aligned}
S_{n} & =\left(T^{h}\right)^{n}-\left(R^{h}\right)^{n} \\
& =\sum_{j=0}^{n-1}\left(T^{h}\right)^{n-1-j}\left(T^{h}-R^{h}\right)\left(R^{h}\right)^{j} .
\end{aligned}
$$

We may as well show that

$S_{n}^{\prime}=\sum_{j=0}^{n-2}\left(T^{h}\right)^{n-1-j}\left(T^{h}-R^{h}\right)\left(R^{h}\right)^{j} \rightarrow 0$, since the omitted term $\left(T^{h}-R^{h}\right)\left(R^{h}\right)^{n-1} \rightarrow(I-I) R^{t}=0$.

Since the $S_{n}^{\prime}$ are uniformly bounded, it is enough to show that $S_{n}^{\prime} u \rightarrow 0$ for a dense set of $u$ in $H$.

Let $u$ be in $D(C)$. Then

$$
\left\|S_{n}^{\prime} u\right\|=\sup \left(v \in H^{1}, v \neq 0\right)\left|\left(S_{n}^{\prime} u, v\right)\right| /\|v\|
$$

since $H^{1}$ is dense in $H$. So if $v \neq 0$ is in $H^{1}$, consider the expression $\left(S_{n}^{\prime} u, v\right)$. If we write $R=R^{h}, T=T^{h}$, and $D=T-R$, we have

$$
\begin{aligned}
\left|\left(S_{n}^{\prime} u, v\right)\right| & \leqq \sum_{j=1}^{n-2}\left|\left(D R^{j} u, T^{* n-1-j} v\right)\right| \\
& \leqq \sum_{j=0}^{n-2}\left\|D R^{j} u\right\|_{-1}\left\|T^{* n-1-j} v\right\|_{1} \\
& \leqq\left(\sum_{j=0}^{n-2}\left\|D R^{j} u\right\|_{-1}^{2}\right)^{1 / 2}\left(\sum_{j=0}^{n-2}\left\|T^{* n-1-j} v\right\|_{1}^{2}\right)^{1 / 2} \\
& =\left(\frac{1}{n} \sum_{j=0}^{n-2}\left\|n D R^{j} u\right\|_{-1}^{2}\right)^{1 / 2}\left(\frac{1}{n} \sum_{j=0}^{n-2}\left\|T^{* n-1-j} v\right\|_{1}^{2}\right)^{1 / 2} .
\end{aligned}
$$

We want to estimate these terms.

Lemma 1. Let $H^{1} \subset H \subset H^{-1}$ and $A$ be as in the theorem. Then $A$ has a restriction to an operator $A^{\prime}$ in $H^{1}$ which generates a semigroup $\exp t A^{\prime}$ on $H^{1}$ which is a restriction of $\exp t A$ on $H$. Both these semigroups are holomorphic for $t>0$.

Proof. $A$ has an extension $\hat{A} \in L\left(H^{1}, H^{-1}\right)$. The norm on $H^{1}$ may be given by $(u, u)_{1}=\operatorname{Re}((-\hat{A}+1) u, u)$. It is sufficient to show that $\hat{A_{1}}=\hat{A}-1$, when restricted to $\left\{u \in H^{1}: \hat{A}_{1} u \in H^{1}\right\}$, is the infinitesimal generator of a holomorphic semigroup. 
Regarding $\hat{A_{1}}$ as in $L\left(H^{1}, H^{-1}\right)$, also $\hat{A}_{1}^{*} \in L\left(H^{1}, H^{-1}\right)$. So we may write $\hat{A}_{1}=\hat{C}+i \hat{D}$ with $\hat{C}, \hat{D}$ self-adjoint in the usual way. Thus $(u, v)_{1}=(-\hat{C} u, v)$.

Consider the operators $\hat{A}_{\alpha}=\hat{C}+i \alpha \hat{D}, 0 \leqq \alpha \leqq 1$. Then also $(u, u)_{1}=-\operatorname{Re}\left(\hat{A}_{\alpha} u, u\right)$.

Now $\hat{D} \in L\left(H^{1}, H^{-1}\right)$, so we can consider the set $N$ of complex numbers $z$ with $|z|=1, \operatorname{Re} z \geqq 3 / 4$ and

$$
|\operatorname{Im} z| \mid \hat{D} \| \leqq 1 / 2 \text {. }
$$

Let $\theta$ be the maximum argument of these numbers.

Let $S$ be a sector in the open left half-plane together with the origin which is bounded by rays making angles less than $\theta$ with the imaginary axis and such that $\hat{A}$ is of type $S$.

Let \|\|$_{1}$ also denote the norm in $L\left(H^{1}\right)$. Consider the set $J$ of all $\alpha$ such that $\hat{A}_{\alpha}$ has a restriction to an operator $A_{\alpha}^{\prime}$ in $H^{1}$ such that for all $\lambda \notin S,\left(\lambda-A_{\alpha}^{\prime}\right)^{-1} \in L\left(H^{1}\right)$ and satisfies

$$
\left\|\left(\lambda-A_{\alpha}^{\prime}\right)^{-1}\right\|_{1} \leqq \frac{M}{d(\lambda, S)}
$$

for some constant $M . \quad(d(\lambda, S)$ is the distance from $\lambda$ to $S) \quad B$. Hille's theory [13, §12.8] (see also Nelson [25]), $\alpha \in J$ implies that $A_{\alpha}$ generates a holomorphic semi-group on $H^{1}$. Thus our main task is to show that $1 \in J$.

The maximal restriction of $\widehat{C}$ to an operator $C^{\prime}$ in $H^{1}$ is clearly symmetric with the inner product $-(\hat{C} u, v)$. Since $\hat{C} \in L\left(H^{1}, H^{-1}\right)$ is easily seen to be onto $H^{-1}$, the maximal restriction $C^{\prime}$ is onto $H^{1}$. This proves self-adjointness. Since $\left(C^{\prime} u, u\right)_{1}=-(\hat{C} u, \hat{C} u), C^{\prime}$ is clearly negative. Thus $0 \in J$.

We now show that $\left(\lambda-\hat{A}_{\alpha}\right)^{-1} \in L\left(H^{-1}, H^{1}\right)$ for $\lambda \in S$. Choose $z$ in $N$ such that $\operatorname{Re} \lambda \bar{z} \geqq 0$. Then

$$
\begin{aligned}
& \operatorname{Re}\left(\left(\lambda-\hat{A}_{\alpha}\right) u, z u\right) \\
& \quad=\operatorname{Re} \lambda \bar{z}(u, u)+\operatorname{Re}\left(\bar{z}\left(-\hat{A}_{\alpha} u, u\right)\right) \\
& \quad \geqq \operatorname{Re}\left(\bar{z}\left(-\hat{A}_{\alpha} u, u\right)\right) \\
& \quad=\operatorname{Re} \bar{z} \operatorname{Re}\left(-\hat{A}_{\alpha} u, u\right)-\operatorname{Im} \bar{z} \operatorname{Im}\left(-\hat{A}_{\alpha} u, u\right) \\
& \quad \geqq 3 / 4(u, u)_{1}-\operatorname{Im} \bar{z} \operatorname{Im}\left(-\hat{A}_{\alpha} u, u\right) \\
& \quad=3 / 4(u, u)_{1}+\alpha \operatorname{Im} \bar{z}(\hat{D} u, u) \\
& \quad \geqq 1 / 4(u, u)_{1}=1 / 4\|u\|_{1}^{2} .
\end{aligned}
$$

The first term above is majorized by $\left\|\left(\lambda-\hat{A}_{\alpha}\right) u\right\|_{-1}\|u\|_{1}$. This shows that $\lambda-\hat{A}_{\alpha}$ is one-to-one and bicontinuous from $H^{1}$ to $H^{-1}$. To show that it is onto $H^{-1}$, suppose otherwise. Then the range isn't even dense, so there is a $v$ in $H^{1}$ with 


$$
\left(\left(\lambda-\hat{A}_{\alpha}\right) u, v\right)=0 \text { for all } u \text { in } H^{1} .
$$

But then in particular

$$
\left(\left(\lambda-\hat{A}_{\alpha}\right) \bar{z} v, v\right)=0 \text {, so by the above }\|v\|_{1}=0 \text {. }
$$

Thus $\left\|\left(\lambda-\hat{A}_{\alpha}\right)^{-1}\right\| \in L\left(H^{-1}, H^{1}\right)$ with norm $\leqq 4$.

Now assume $\alpha \in J$. Choose $\varepsilon$ with $\varepsilon\|\hat{D}\|<1 / 8$. Then

$$
\begin{aligned}
\| & \left(\lambda-A_{\alpha+\varepsilon}^{\prime}\right)^{-1} \|_{1} \\
& =\left\|\left(\lambda-A_{\alpha}^{\prime}-i \varepsilon D^{\prime}\right)^{-}\right\|_{1} \\
& \leqq\left\|\left(\lambda-A_{\alpha}^{\prime}\right)^{-1}\right\|_{1}\left\|\left(1-i \varepsilon \hat{D}\left(\lambda-\hat{A}_{\alpha}\right)^{-1}\right)^{-1}\right\|_{1} \\
& \leqq \frac{M}{d(\lambda, \mathscr{S})} \cdot 2 .
\end{aligned}
$$

Thus $\alpha+\varepsilon$ is in $J$. This is sufficient to show $1 \in J$.

Hille's theory gives an explicit expression for the holomorphic semigroup as an inverse Laplace transform contour integral. Thus as an integral in the strong topology of $H^{1}$

$$
\exp t A^{\prime}=\frac{1}{2 \pi i} \int_{\Gamma} e^{t \lambda}\left(\lambda-A^{\prime}\right)^{-1} d \lambda \text {. }
$$

The integral clearly converges also in the strong topology of $H$, and since this formula is also valid for $A$ in $H$, the semigroup in $H^{1}$ must be just a restriction of the one in $H$.

Lemma 2. Let $H^{1} \subset H \subset H^{-1}$ and $A$ be as in the theorem. Let $P^{t}=\exp t A . \quad$ Let $Q^{t}$ be any contraction semigroup on $H$. Then

$$
\frac{1}{n} \sum_{k=1}^{n}\left\|\left(P^{h} Q^{h}\right)^{k} v\right\|_{1}^{2}
$$

(where $h=t / n)$ is bounded by $\|v\|^{2}$ times a constant independent of $n$.

Proof. First of all, note that $R\left(P^{t}\right) \subset D(A) \subset H^{1}$ for all $t>0$.

The formula by which an operator $A$ of type $S$ may be used to determine a norm on $H^{1}$ gives

$$
\left\|P^{t} w\right\|_{1}^{2}=-\operatorname{Re}\left(A P^{t} w, P^{t} w\right)+\left\|P^{t} w\right\|^{2} .
$$

(This step is where the proof fails for the Schrödinger equation.) Also,

$$
\frac{d}{d t} \frac{1}{2}\left\|P^{t} w\right\|^{2}=\operatorname{Re}\left(A P^{t} w, P^{t} w\right)
$$

These two combine to give the crucial estimate 


$$
\left\|P^{t} w\right\|_{1}^{2}=-\frac{1}{2} \frac{d}{d t}\left\|P^{t} w\right\|^{2}+\left\|P^{t} w\right\|^{2}
$$

Integrating,

$$
\int_{0}^{h}\left\|P^{s} w\right\|_{1}^{2} d s=\frac{1}{2}\|w\|^{2}-\frac{1}{2}\left\|P^{h} w\right\|^{2}+\int_{0}^{h}\left\|P^{s} w\right\|^{2} d s .
$$

Now since any strongly continuous semigroup is bounded on finite time intervals, we have by Lemma 1 for $0<s \leqq h$

$$
\begin{aligned}
\left\|P^{h} w\right\|_{1} & \leqq\left\|P^{h-s}\right\|_{1}\left\|P^{s} w\right\|_{1} \\
& \leqq M\left\|P^{s} w\right\|_{1} .
\end{aligned}
$$

Hence $h\left\|P^{h} w\right\|_{1}^{2} \leqq M^{2} \int_{0}^{h}\left\|P^{s} w\right\|_{1}^{2} d s$.

So the estimate becomes

$$
h\left\|P^{h} w\right\|_{1}^{2} \leqq M^{2}\left(\frac{1}{2}\|w\|^{2}-\frac{1}{2}\left\|P^{h} w\right\|^{2}+h\|w\|^{2}\right) .
$$

Now apply this to $w$ of the form $Q^{h}\left(P^{h} Q^{h}\right)^{k-1} v$, where $1 \leqq k \leqq n-1$, and add. Thus

$$
\begin{aligned}
\sum_{k=1}^{n-1} h & \left\|\left(P^{h} Q^{h}\right)^{k} v\right\|_{1}^{2} \\
\leqq & M^{2}\left\{\frac{1}{2} \sum_{k=1}^{n-1}\left(\left\|Q^{h}\left(P^{h} Q^{h}\right)^{k-1} v\right\|^{2}-\left\|\left(P^{h} Q^{h}\right)^{k} v\right\|^{2}\right)\right. \\
& \left.+h \sum_{k=1}^{n-1}\left\|Q^{h}\left(P^{h} Q^{h}\right)^{k-1} v\right\|^{2}\right\} \\
\leqq & M^{2}\left\{\frac{1}{2} \sum_{k=1}^{n-1}\left(\left\|\left(P^{h} Q^{h}\right)^{k-1} v\right\|^{2}-\left\|\left(P^{h} Q^{h}\right)^{k} v\right\|^{2}\right)\right. \\
& \left.+t / n \sum_{k=1}^{n-1}\|v\|^{2}\right\} \\
\leqq & M^{2}\left\{\frac{1}{2}\left(\|v\|^{2}-\left\|\left(P^{h} Q^{h}\right)^{n-1} v\right\|^{2}\right)+t\|v\|^{2}\right\} \\
\leqq & M^{2}\left(\frac{1}{2}+t\right)\|v\|^{2} .
\end{aligned}
$$

This proves the lemma.

Now note that with $\hat{A} \in L\left(H^{1}, H^{-1}\right)$ we have $\hat{A}^{*} \in L\left(H^{1}, H^{-1}\right)$ and that $(u, v)_{1}=((-\operatorname{Re} \hat{A}+1) u, v)=\left(\left(-\operatorname{Re} \hat{A}^{*}+1\right) u, v\right)$. Next observe that in particular when $u \in D(A)$ and $\hat{A}^{*} v \in H$, then $(A u, v)=\left(u, \hat{A}^{*} v\right)$, so that the maximal restriction of $\hat{A}^{*}$ is just the adjoint of $A$ as an operator on $H$. Now since Hilbert space is reflexive, the infinitesimal 
generator of $P^{t^{*}}$ on $H$ is just this operator $A^{*}$.

Now apply Lemma 2 to $1 / n \sum_{j=0}^{n-2}\left\|\left(P^{h^{*}} Q^{h^{*}}\right)^{n-1-j} v\right\|_{1}^{2}$, noticing that the norm $\operatorname{Re}\left(-\hat{A}^{*} u, u\right)+(u, u)$ used in the lemma is equal to the norm $\|u\|_{1}^{2}=\operatorname{Re}(-\hat{A} u, u)+(u, u)$ occurring in this expression. This bounds $1 / n \sum_{j=0}^{n-2}\left\|T^{* n-1-j} v\right\|_{1}^{2}$ by a constant times $\|v\|$.

Now consider the other term

$$
\begin{aligned}
& \frac{1}{n} \sum_{j=1}^{n-2}\left\|n D^{h} R^{h j} u\right\|_{-1}^{2} \\
& \quad \leqq \max (0 \leqq j \leqq n-2)\left\|n D^{h} R^{h j} u\right\|_{-1}^{2} \\
& \quad \leqq \sup (0 \leqq s \leqq t)\left\|n D^{h} R^{s} u\right\|_{-1}^{2} .
\end{aligned}
$$

Let $E$ be the set of all $R^{s} u, 0 \leqq s \leqq t$. Note that $E \subset D(C) \subset H^{1}$. It is sufficient to show that

$$
\left\|n D^{h} w\right\|_{-1} \rightarrow 0
$$

uniformly on $w$ in $E$. Rewrite this as $t$ times

$$
\begin{aligned}
& \left\|\frac{1}{h}\left(Q^{h} P^{h}-R^{h}\right) w\right\|_{-1} \\
& =\left\|\frac{1}{h}\left(P^{h}-1\right) w+\frac{1}{h}\left(Q^{h}-1\right) P^{h} w-\frac{1}{h}\left(R^{h}-1\right) w\right\|_{-1} .
\end{aligned}
$$

First consider the term $1 / h\left(P^{h}-1\right) w$. We have $1 / h\left(P^{h}-1\right) w=$ $1 / h \int_{0}^{h} \hat{A} P^{s} w d s$ certainly for $w$ in $D(A)$. Since these $P^{s}, 0 \leqq s \leqq h$, are uniformly bounded in $L\left(H^{1}\right)$ (by Lemma 1), the $\hat{A} P^{s}$ are uniformly bounded in $L\left(H^{1}, H^{-1}\right)$. Since $s \rightarrow \widehat{A} P^{s} w$ is continuous for $w$ in $D(A)$, which is dense in $H^{1}$, it is continuous for all $w$ in $H^{1}$. Hence $1 / h \int_{0}^{h} \hat{A} P^{s} w d s$ is defined for all $w$ in $H^{1}$, and we have $1 / h\left(P^{h}-1\right)=$ $1 / h \int_{0}^{h} \hat{A} P^{s} d s$, since both sides are bounded $H^{1} \rightarrow H^{-1}$ and they are equal on a dense set. Hence

$$
\begin{aligned}
& \left\|\frac{1}{h}\left(P^{h}-1\right) w-\hat{A} w\right\|_{-1} \\
& \quad=\left\|\frac{1}{h} \int_{0}^{h}\left(\hat{A} P^{s} w-\hat{A} w\right) d s\right\|_{-1} \\
& \quad \leqq \sup (0 \leqq s \leqq h)\left\|\hat{A} P^{s} w-\hat{A} w\right\|_{-1} .
\end{aligned}
$$

But $\hat{A} P^{h} w=P^{h} \hat{A} w \rightarrow \hat{A} w$ as $h \rightarrow 0$. So $1 / h\left(P^{h}-1\right) w \rightarrow \hat{A} w$ in $H^{-1}$ for all $w$ in $H^{1}$.

Similarly, $1 / h\left(R^{h}-1\right) w \rightarrow \hat{C} w$ in $H^{-1}$. (Here Lemma 1 is applied to $C-1$ in order to get the required bound on $R^{s}$.) 
Now examine $1 / h\left(Q^{h}-1\right) P^{h} w$. For $w$ in $D(B) \cap H^{1}, 1 / h\left(Q^{h}-1\right) w=$ $-1 / h \int_{0}^{h}(-B)^{1 / 2} Q^{s}(-B)^{1 / 2} w d s$.

Now $(-B)^{1 / 2}$ was assumed to be bounded from a dense subspace of $H^{1}$ to $H$, and so has an extension to $(-\widehat{B})^{1 / 2}: H^{1} \rightarrow H$.

$(-B)^{1 / 2}$ is also continuous from a dense subspace of $H$ to $H^{-1}$. For if $u$ and $v$ are in the appropriate subspaces of $D\left((-B)^{1 / 2}\right)$ and $D\left((-B)^{1 / 2^{*}}\right)$,

$$
\begin{aligned}
& \left|\left((-B)^{1 / 2} u, v\right)\right|=\left|\left(u,(-B)^{1 / 2^{*}} v\right)\right| \\
& \quad \leqq\|u\|\left\|(-B)^{1 / 2^{*}} v\right\| \leqq M\|u\|\|v\|_{1} .
\end{aligned}
$$

So we also get a bounded extension $(-\hat{B})^{1 / 2}: H \rightarrow H^{-1}$.

The product $(-\hat{B})^{1 / 2}(-\widehat{B})^{1 / 2}$ of these two extensions is a bounded operator which agrees with $-\hat{B}: H^{1} \rightarrow H^{-1}$.

$(-\hat{B})^{1 / 2} Q^{s}(-\hat{B})^{1 / 2} w$ is continuous in $H^{-1}$ for all $w$ in $H^{1}$, so the integral

$$
\frac{1}{h} \int_{0}^{h}(-\hat{B})^{1 / 2} Q^{s}(-\hat{B})^{1 / 2} w d s \text { exists for all } w \text { in } H^{1}
$$

$1 / h\left(Q^{h}-1\right)=-1 / h \int_{0}^{h}(-\hat{B})^{1 / 2} Q^{s}(-\hat{B})^{1 / 2} d s$ since both sides are bounded from $H^{1}$ to $H^{-1}$ and agree on a dense subspace. (This is the only place where the assumption that $D(B)$ is dense in $H^{1}$ is used.)

As before,

$$
\begin{aligned}
& \left\|\frac{1}{h}\left(Q^{h}-1\right) w-\hat{B} w\right\|_{-1} \\
& \quad=\left\|\frac{1}{h} \int_{0}^{h}\left(-(-\hat{B})^{1 / 2} Q^{s}(-\hat{B})^{1 / 2} w-\hat{B} w\right) d s\right\|_{-1} \\
& \quad \leqq \sup (0 \leqq s \leqq h) !-(-\hat{B})^{1 / 2} Q^{s}(-\hat{B})^{1 / 2} w-\hat{B} w \|_{-1} .
\end{aligned}
$$

So $1 / h\left(Q^{h}-1\right) w \rightarrow \hat{B} w$ in $H^{-1}$.

Now $t \rightarrow P^{t} w$ is continuous in $H^{1}$ for all $w$ in $D(A)$, and so, $P^{t}$ being bounded on $H^{1}$ by Lemma $1, t \rightarrow P^{t} w$ is continuous in $H^{1}$ for all $w$ in $H^{1}$.

$1 / h\left(Q^{h}-1\right) w$ is bounded in $H^{-1}$ for each $w$ in $H^{1}$; hence the norms are bounded, by the principle of uniform boundedness.

It follows from these remarks that

$$
\frac{1}{h}\left(Q^{h}-1\right) P^{h} w \rightarrow \hat{B} w
$$

Now $1 / h D^{h} w=1 / h\left(P^{h}-1\right) w+1 / h\left(Q^{h}-1\right) P^{h} w-1 / h\left(R^{h}-1\right) w=$ $1 / h\left(P^{h}-1\right) w-\hat{A} w+1 / h\left(Q^{h}-1\right) P^{h} w-\hat{B} w-1 / h\left(R^{h}-1\right) w+\hat{C} w \rightarrow 0$ 
in $H^{-1}$ for each $w$ in $H^{1}$. Hence, by the principle of uniform boundedness, the norms of the $1 / h D^{h}: H^{1} \rightarrow H^{-1}$ are uniformly bounded.

Since the norm in $H^{1}$ could just as well be defined by $\hat{C}$, and $C R^{s} u=R^{s} C u, s \rightarrow R^{s} u \in H^{1}$ is continuous in $s$. Thus $E$ is the continuous image of $[0, t]$, hence compact.

Hence $1 / h D^{h} w \rightarrow 0$ in $H^{-1}$ uniformly on $w$ in $E$.

3. Continuity in the mass parameter. The Schrödinger equation involves $(i / 2 \mathrm{~m}) \Delta$, which does not have its spectrum in a sector bounded by rays in the open left half-plane. Thus it should not be regarded as a parabolic equation. However, we can use the product formula previously derived for parabolic equations to define the Feynman integral solution of the Schrödinger equation by employing a continuation in the mass parameter.

The solution to the time-independent Schrodinger equation with mass $m>0$ is (formally) the unitary operator $\exp ((i / 2 m) \Delta-i V) t$. Continuation to the parabolic diffusion equation in the parameter $t$ has the effect of replacing $t$ by $-i t^{\prime}$ in the exponent to get $((1 / 2 m) \Delta-V) t^{\prime}$. The Trotter product representation of the solution of this equation involves the expression $\exp \left(-V t^{\prime} / n\right)$. This is a bounded operator on $L^{2}$ only when $V$ is bounded below. Even when this condition doesn't hold, the sum $(-1 / 2 m) \Delta+V$ may be bounded below, so that a solution (perhaps defined using a Friedrichs extension) should exist. However, the preceding $L^{2}$ approach to a product representation of the solution seems difficult to apply. Babbit [2] evades this obstacle in his representation of the solution of the diffusion equation as a Wiener integral of an unbounded function.

The advantage of continuation in the parameter $m$ is that replacing $m$ by $i m^{\prime}, m^{\prime}>0$, we get $\left(\left(1 / 2 m^{\prime}\right) \Delta-i V\right) t$ and each term always has negative real part, no matter how singular $V$ is. The disadvantage, of course, is that the parameter $m$ is buried more deeply in the equation.

The following result on continuity in a parameter will be needed later for this application to the Schrödinger equation.

Theorem 2. Let $A$ be the infinitesimal generator of a contraction semigroup in $H$. Assume that a complex number $\sigma$ with $|\sigma|=1$ may be chosen so that $\sigma A$ is of type $S$, where $S-\{0\}$ is contained in the open left half-plane. Let $H^{1} \subset H \subset H^{-1}$ be the scale associated with $\sigma A$. Let $\alpha$ be any complex number such that $\alpha A$ is dissipative.

Let $\hat{B}$ be a dissipative operator in $L\left(H^{1}, H^{-1}\right)$. Assume that some norm for $H^{1}$ of the form $\|u\|_{1}^{2}=\operatorname{Re}((-\sigma \hat{A}+k) u, u)$ may be chosen so that the operator norm of $\hat{B}$ is then strictly less than $|\alpha|$. Then $\alpha \hat{A}+\hat{B} \in L\left(H^{1}, H^{-1}\right)$ has a restriction to an operator $C_{\alpha}$ 
which generates a contraction semigroup $\exp \left(t C_{\alpha}\right), \exp \left(t C_{\alpha}\right)$ is strongly continuous in $\alpha$ for fixed $t$.

$$
\begin{aligned}
\text { Proof. } & \text { For } \lambda>0, \\
\left|\left(\left(\lambda-\hat{C}_{\alpha}\right) u, u\right)\right| & |((\lambda-\alpha \hat{A}) u, u)|-|(\hat{B} u, u)| \\
\geqq & |((\lambda-\alpha \hat{A}) u, u)|-\|\hat{B}\|\|u\|_{1}^{2} \\
\geqq & \left(\frac{\|\hat{B}\|+\varepsilon}{|\alpha|}+\frac{\varepsilon}{|\alpha|}\right)|\lambda(u, u)+(-\alpha \hat{A} u, u)| \\
& -\|\hat{B}\|[|(\hat{A} u, u)|+k(u, u)] \\
& (\text { where } \varepsilon>0 \text { is such that }\|\hat{B}\|+2 \varepsilon=|\alpha|) \\
\geqq & (\|\hat{B}\|+\varepsilon)|(A u, u)|+\lambda \frac{\varepsilon}{|\alpha|}(u, u) \\
& -\|\hat{B}\||(\hat{A} u, u)|-\|\hat{B}\| k(u, u) \\
& (\operatorname{since} \operatorname{Re}(-\alpha \hat{A} u, u) \geqq 0) \\
= & \varepsilon|(\hat{A} u, u)|+\left(\lambda \frac{\varepsilon}{|\alpha|}-\|\hat{B}\| k\right)(u, u) \\
\geqq & c \operatorname{Re}((k-\sigma \hat{A}) u, u)=c\|u\|_{1}^{2}
\end{aligned}
$$

for some $c>0$ and $\lambda$ sufficiently large.

Thus the discussion of the Friedrichs extension shows that $\left(\lambda-\hat{C}_{\alpha}\right)^{-1} \in L\left(H^{-1}, H^{1}\right)$ and that the restricted $C_{\alpha}$ generates a semigroup $\exp t C_{\alpha}$.

Now $\left(\lambda-\hat{C}_{\alpha}\right)^{-1}$ is in fact continuous in the norm topology of $L\left(H^{-1}, H^{1}\right)$. For

$$
\begin{aligned}
\left(\lambda-\widehat{C}_{\alpha^{\prime}}\right)^{-1}= & \left(1-\left(\lambda-\widehat{C}_{\alpha}\right)^{-1}\left(\widehat{C}_{\alpha^{\prime}}-\widehat{C}_{\alpha}\right)\right)^{-1}\left(\lambda-\widehat{C}_{\alpha}\right)^{-1} \\
& \rightarrow\left(\lambda-\widehat{C}_{\alpha}\right)^{-1} \text { as } \alpha \rightarrow \alpha^{\prime} .
\end{aligned}
$$

Therefore $\left(\lambda-C_{\alpha}\right)^{-1}$ is continuous in the norm topology of $L(H)$. Therefore it is continuous in the strong topology of $L(H)$. Theorem 5.1 and Lemma 5.1 of Trotter [27] (see also Nelson [25], Yosida [29]) imply the desired result.

4. Multiplication operators on Sobolev spaces. This section illustrates the kind of estimates needed to apply the Friedrichs method in concrete situations. These show in what sense certain terms in a differential operator may be regarded as small perturbations. The estimates presented here compare multiplicative terms (zeroth order differential operators) with the Laplacian. The same techniques may be used to obtain results about perturbations of elliptic operators of arbitrary order by lower order differential operators. 
We start with the Hilbert space $H=L^{2}\left(\boldsymbol{R}^{l}\right)$. The Laplace operator $\Delta$ is initially regarded as a self-adjoint operator in $H$. The real valued measurable function $V$ acts as a multiplication operator.

Let $H^{2}$ be the space of functions in $L^{2}\left(\boldsymbol{R}^{l}\right)$ with second partial derivatives in $L^{2}\left(\boldsymbol{R}^{l}\right)$. Then $H^{2}=D(\Delta)$. Assume that $V$ is bounded from $H^{2}$ to $H$, that is, $V \in L\left(H^{2}, H\right)$. This means that there are $a>0, b>0$ such that

$$
\|V u\| \leqq a\|\Delta u\|+b\|u\|,
$$

for all $u$ in $D(\Delta)$. If for all $a>0$ there exists $b>0$ such that this holds, then $V$ will be said to be strictly bounded from $H^{2}$ to $H$. (This is what Nelson [24] calls a Kato potential.)

Now $\Delta$ is of type the negative real axis, so the Friedrichs method is applicable. Let $H^{1} \subset H \subset H^{-1}$ be the scale associated with $\Delta$. Then $H^{1}$ is the space of functions in $L^{2}\left(\boldsymbol{R}^{l}\right)$ with first partial derivatives in $L^{2}\left(\boldsymbol{R}^{l}\right)$. In fact $H^{1}=D\left((-\Delta)^{1 / 2}\right) . \quad H^{-1}$ is a certain space of distributions on $\boldsymbol{R}^{l}$. It will later be useful to interpret the elements of $H^{-1}$ as distributions of some kind of charge. A norm for $H^{-1}$ may be regarded as giving the energy of self-interaction of this type of charge. With this notion of energy, $H^{-1}$ is the space of distributions of finite energy.

Thus we have $H^{2} \subset H^{1} \subset H \subset H^{-1} . \Delta$ may now be considered also as an operator in $L\left(H^{2}, H\right)$ or in $L\left(H^{1}, H^{-1}\right)$.

Assume that we want to show that $V$ on $H^{1}$ is bounded from all of $H^{1}$ to $H^{-1}$. It is enough (Lang [18, p. 114]) to show that there are $a>0, b>0$ such that

$$
|(V u, u)| \leqq a(-\Delta u, u)+b(u, u), u \in H^{1} .
$$

If for all $a>0$ there is a $b>0$ with this holding, then $V$ will be termed strictly bounded from $H^{1}$ to $H^{-1}$.

Proposition 4. $V \in L^{r}\left(\boldsymbol{R}^{l}\right), r \geqq l / 2, r \geqq 2$ implies $V$ is strictly bounded from $H^{2}$ to $H$. Exception: $l=2 r=4$.

Proof. Here it will be convenient to write $\|u\|_{p}$ for the norm of $L^{p}\left(\boldsymbol{R}^{l}\right)$.

Case 1. $r>l / 2$.

Let $u \in D(\Delta)$. Let $1 / r+1 / s=1 / 2$. Then $1 / r \leqq 1 / 2$ implies $0 \leqq 1 / s$, so

$$
\|V u\|_{2} \leqq\|V\|_{r}\|u\|_{s} \text {. }
$$

Let $\hat{u}$ be the Fourier transform of $u$. If $1 / s+1 / q=1$, then 
$0 \leqq r$ implies $1 / s \leqq 1 / 2$, which implies that $1 / 2 \leqq 1 / q$. So $1 / q$ is in the range $[1 / 2,1]$, and so

$$
\|u\|_{s} \leqq\|\hat{u}\|_{q}
$$

by the Hausdorff-Young theorem (Nelson [25]).

Now let $k$ be a real parameter. Then

$$
\begin{aligned}
\|\hat{u}\|_{q} & =\left\|\frac{1}{1+k^{2}|x|^{2}}\left(1+k^{2}|x|^{2}\right) \hat{u}\right\|_{q} \\
& \leqq\left\|\frac{1}{1+k^{2}|x|^{2}}\right\|_{r}\left\|\left(1+k^{2}|x|^{2}\right) \hat{u}\right\|_{2}
\end{aligned}
$$

since $1 / r+1 / 2=1-1 / s=1 / q$.

The first factor is finite since $l<2 r$. Thus

$$
\begin{aligned}
\|u\|_{q} & \leqq \frac{C}{k^{l / r}}\left(\|\hat{u}\|_{2}+k^{2}\left\|\left.x\right|^{2} \widehat{u}\right\|_{2}\right) \\
& =\frac{C}{k^{l / r}}\left(\|u\|_{2}+k^{2} \mid\left\langle u \|_{2}\right)\right.
\end{aligned}
$$

be the Plancherel theorem.

This may be summarized (with modifications in the case $r=\infty$ ):

$$
\|u\|_{s} \leqq C k^{l / r(r-l / 2)}\|\Delta u\|_{2}+C k^{-l / r}\|u\|_{2} .
$$

The conclusion follows from (1) and (2).

Case 2. $r=l / 2, l \neq 4$. (Notice that this case is significant only for $l>4$.)

Let $\chi_{N}$ be the characteristic function of $\left\{x \in R^{l},|V(x)| \leqq N\right\}$. Let $V_{N}=\chi_{N} V, V_{N}^{\prime}=\left(1-\chi_{N}\right) V$. Then

$$
\|V u\|_{2} \leqq\left\|V_{N} u\right\|_{2}+\left\|V_{N}^{\prime} u\right\|_{2} \text {. }
$$

Clearly $\left\|V_{N} u\right\|_{2} \leqq\left\|V_{N}\right\|_{\infty}\|u\|_{2} \leqq N\|u\|_{2}$.

On the other hand, we have assumed that $1 / r=21 / l$ and $1 / r \leqq 2$. Thus if $1 / r+1 / s=1 / 2,1 / s \geqq 0$, so

$$
\left\|V_{N}^{\prime} u\right\|_{2} \leqq\left\|V_{N}^{\prime}\right\|_{r}\|u\|_{s} \text {. }
$$

$V_{N}^{\prime}$ is also in $L^{r}$ and in fact $\left|V_{N}^{\prime}\right|^{r}$ is dominated by $|V|^{r}$. Hence by the dominated convergence theorem, $\left\|V_{N}^{\prime}\right\|_{r} \rightarrow 0$ as $N \rightarrow \infty$.

Now the Sobolev inequality (Nirenberg [26]) says that

$$
\|u\|_{k} \leqq C\|D u\|_{p} \text { where } 1 / k=1 / p-1 / l \text { and } 1 / l<1 / p .
$$

Here $\|D u\|_{p}$ represents $\left\|\left(\partial u / \partial x_{1}\right)\right\|_{p}+\cdots+\left\|\left(\partial u / \partial x^{l}\right)\right\|_{p}$. 
Now since $l \neq 4,1 / r<1 / 2$, and $1 / s>0$. Letting $1 / q=1 / s+1 / l$, the inequality shows that

$$
\|u\|_{s} \leqq C\|D u\|_{q}, \text { since } 1 / s=1 / q-1 / l>0
$$

and that

$$
\begin{aligned}
\|w\|_{q} & \leqq C\|D w\|_{2}, \text { since } 1 / q=1 / s+1 / l \\
& =1 / 2-1 / r+1 / l=1 / 2-21 / l+1 / l \\
& =1 / 2-1 / l .
\end{aligned}
$$

It follows that

$$
\|u\|_{s} \leqq C\|\Delta u\|_{2} \text {. }
$$

In the case of the Schrödinger equation in dimensions $l=1,2,3$ the condition $r \geqq 2$ is an additional restriction. If we replace this by $r \geqq 1$ we get only the following result.

Proposition 5. Let $V \in L^{r}\left(\boldsymbol{R}^{l}\right), r \geqq l / 2$ (and of course $r \geqq 1$ ). Then $V$ is strictly bounded from $H^{1}$ to $H^{-1}$. Exception: $l=2 r=2$.

Case 1. $r>l / 2$.

This follows from the lemma:

Lemma. Let $W \in L^{p}\left(R^{l}\right), p>l, p>2$. Then for all $a>0$ there is $a b>0$ so that

$$
\|W u\|_{2} \leqq a\|\sqrt{-\Delta} u\|_{2}+b\|u\|_{2} \text {. }
$$

Proof. $1 / p<1 / l$ and $1 / p \leqq 1 / 2$.

If $1 / p+1 / t=1 / 2$, then $1 / p \leqq 1 / 2$ implies $t$ is positive, and so

$$
\|W u\|_{2} \leqq\|W\|_{p}\|u\|_{t} \text {. }
$$

Next we must show

$$
\|u\|_{t} \leqq a\|\sqrt{-\Delta} u\|_{2}+b\|u\|_{2} .
$$

The proof is almost the same as before. It uses the Hausdorff-Young theorem; now the crucial fact is that $l<p$.

Case 2. $r=l / 2, l>2$.

As before, write $V=V_{N}+V_{N}^{\prime}$. Then $\left|\left(V_{N} u, u\right)\right| \leqq N(u, u)$.

We have $1 / r=21 / l$ and $1 / l<1 / 2$.

$$
\begin{aligned}
\left|\left(V_{N}^{\prime} u, u\right)\right| & \leqq\left\|\sqrt{\overline{V_{N}^{\prime}} \mid} u\right\|_{2}^{2} \\
& \leqq\left\|\sqrt{\left|V_{N}^{\prime}\right|}\right\|_{i l}^{2}\|u\|_{t}^{2}
\end{aligned}
$$


where $1 / l+1 / t=1 / 2$.

But $\|u\|_{t} \leqq C\|\sqrt{-\Delta} u\|_{2}$ by the Sobolev inequalities, since $1 / t=$ $1 / 2-1 / l$ and $1 / l<1 / 2$.

Proposition 6. Let $H=L^{2}\left(R^{l}\right)$. Assume $l \geqq 3$. Let $g$ be a bounded measurable function on $R^{l}$. Let $r=|x|, x \in \boldsymbol{R}^{l}$. Then as a multiplication operator $g / r^{2}$ is bounded from $H^{1}$ to $H^{-1}$.

Proof. To avoid confusion in this proof, the $L^{p}$ norm on $m$ derivatives is written \|\|$_{m, p}$ following Lions [19].

Let $u$ be a real-valued function in $C_{0}^{\infty}\left(R^{l}\right)$. Regarding $u$ as expressed in spherical polar coordinates and fixing the angle variables $\omega, u(r, \omega)$ is a $C_{0}^{\infty}$ function of $r$. Thus since $l \geqq 3$

$$
\begin{aligned}
& \int_{0}^{\infty} u^{2} r^{l-3} d r=\int_{0}^{\infty} 2 u u^{\prime} \frac{1}{l-2} r^{l-2} d r \\
& =\frac{2}{l-2} \int_{0}^{\infty}\left(u r^{(l-3 / 2)}\right)\left(u^{\prime} r^{(l-1 / 2)}\right) d r \\
& \quad \leqq \frac{2}{l-2}\left[\int_{0}^{\infty} u^{2} r^{l-3} d r\right]^{1 / 2}\left[\int_{0}^{\infty} u^{\prime 2} r^{l-1} d r\right]^{1 / 2} .
\end{aligned}
$$

Therefore

$$
\int_{0}^{\infty} u^{2} r^{l-3} d r \leqq\left(\frac{2}{l-2}\right)^{2} \int_{0}^{\infty} u^{\prime 2} r^{l-1} d r
$$

Now integrating over all the angle variables,

$$
\iint_{0}^{\infty} u^{2} r^{l-3} d r d \omega \leqq\left(\frac{2}{l-2}\right)^{2} \iint_{0}^{\infty}\left(\frac{d u}{d r}\right)^{2} r^{l-1} d r d \omega,
$$

Or $\int u^{2} r^{-2} d x \leqq(2 / l-2)^{2} \int \nabla u \cdot \nabla u d x$.

Now let $u$ be arbitrary in $C_{0}^{\infty}\left(R^{l}\right)$. Writing $|u|^{2}=(\operatorname{Re} u)^{2}+(\operatorname{Im} u)^{2}$, we easily see that

$$
\int|u|^{2} r^{-2} d x \leqq\left(\frac{2}{l-2}\right)^{2} \int \nabla u \cdot \bar{\nabla} u d x
$$

Thus $\|1 / r u\|_{0,2} \leqq(2 / l-2)\|u\|_{1,2}$.

Since such $u$ are dense in $H^{1}$, this is true for all $u$ in $H^{1}$.

If $v$ also is in $H^{1}$, 


$$
\begin{aligned}
\left|\left(\frac{g}{r^{2}} u, v\right)\right| & =\left|\left(\frac{g}{r} u, \frac{1}{r} v\right)\right| \\
& \leqq\left\|\frac{g}{r} u\right\|_{0,2}\left\|\frac{1}{r} v\right\|_{0,2} \\
& \leqq\|g\|_{0, \infty}\left\|\frac{1}{r} u\right\|_{c, 2}\left\|\frac{1}{r} v\right\|_{0,2} \\
& \leqq\|g\|_{0, \infty}\left(\frac{2}{l-2}\right)^{2}\|u\|_{1,2}\|u\|_{1,2} .
\end{aligned}
$$

Hence $\left\|g / r^{2} u\right\|_{-1,2} \leqq\|g\|_{0, \infty}(2 / l-2)^{2}\|u\|_{1,2}$.

Proposition 7. Let $H=L^{2}\left(R^{l}\right)$. Assume $l \geqq 5$. Let $g$ be a bounded measurable function. Let $r=|x|, x \in \boldsymbol{R}^{l}$. Then as a multiplication operator $g / r^{2}$ is bounded from $H^{2}$ to $H$.

Proof. Let $u$ be a real-valued function in $C_{0}^{\infty}\left(R^{l}\right)$. Regarding $u$ as expressed in spherical polar coordinates and fixing the angle variable $\omega, u(r, \omega)$ is a $C_{0}^{\infty}$ function of $r$. Thus

$$
\begin{aligned}
\int u^{2} r^{l-5} d r & \leqq \int 2 u u^{\prime} \frac{1}{l-4} r^{l-4} d r \\
& =\frac{2}{l-4} \int u r^{(l-5) / 2} u^{\prime} r^{(l-3) / 2} d r \\
& \leqq \frac{2}{l-4}\left[\int u^{2} r^{l-5} d r\right]^{1 / 2}\left[\int u^{\prime 2} r^{l-3} d r\right]^{1 / 2}
\end{aligned}
$$

So $\left[\int u^{2} r^{l-5} d r\right]^{1 / 2} \leqq(2 / l-4)\left[\int u^{\prime 2} r^{l-3} d r\right]^{1 / 2}$.

Now as in the previous proof

$$
\left[\int u^{\prime 2} r^{l-3} d r\right]^{1 / 2} \leqq \frac{2}{l-2}\left[\int u^{\prime 2} r^{l-1} d r\right]^{1 / 2}
$$

Hence $\left[\int u^{2} r^{l-5} d r\right]^{1 / 2} \leqq(4 /(l-2)(l-4))\left[\int u^{\prime 2} r^{l-1} d r\right]^{1 / 2}$.

The proof is concluded much as before, giving finally

$$
\left\|\frac{g}{r^{2}} u\right\|_{0,2} \leqq\|g\|_{0, \infty} \frac{4}{(l-2)(l-4)}\|u\|_{2,2} \text {. }
$$

5. Application to the Schrödinger equation. For each $t$ let $u(t)$ be in $H=L^{2}\left(\boldsymbol{R}^{l}\right) . \quad \Delta$ is the Laplace operator and $V$ is a real valued measurable function on $\boldsymbol{R}^{l}$. The Schrödinger equation may be written 


$$
i \frac{d u}{d t}(t)=\left(-\frac{1}{2 m} \Delta+V\right) u(t),
$$

where $m$ is a positive real number (the mass). The solution of the initial value problem should be a one-parameter unitary group on $H$ with $-i(-(1 / 2 m) \Delta+V)$ as its infinitesimal generator. Thus we shall first investigate conditions on $V$ which guarantee that the operator sum $-(1 / 2 m) \Delta+V$ is self-adjoint, or may be interpreted via the Friedrichs method as a self-adjoint operator.

From the preceding estimates we see that if $V$ is a finite sum of functions in various $L^{p}$ spaces; with $p \geqq l / 2, p \geqq 2$ (but $p>2$ when $l=4$ ), then $V$ is strictly bounded from $H^{2}$ to $H$. If $l \geqq 5$ and $V=g / r^{2}$, where $g \in L^{\infty}\left(\boldsymbol{R}^{l}\right)$ with sufficiently small bound, then $V$ is bounded from $H^{2}$ to $H$ with norm less than the norm of $(1 / 2 m) \Delta$. Thus in either case we have

$$
\left(\lambda-\frac{1}{2 m} \Delta+V\right)^{-1}=\left(\lambda-\frac{1}{2 m} \Delta\right)^{-1}\left(1+V\left(\lambda-\frac{1}{2 m} \Delta\right)^{-1}\right)^{-1}
$$

in $L(H)$ for $\lambda$ sufficiently large, since the right hand side may be expanded in a geometric series in $V(\lambda-(1 / 2 m) \Delta)^{-1}$ converging in the norm of $L(H)$. Thus the sum $-(1 / 2 m) \Delta+V$ of these two unbounded self-adjoint operators in $H$ is self-adjoint. This is the situation first studied by Kato [17] in connection with the Schrödinger equation.

If $V$ is a finite sum of functions in various $L^{p}$ spaces, $p \geqq l / 2$, $p \geqq 1$ (but $p>1$ when $l=2$ ), then $V$ is strictly bounded from $H^{1}$ to $H^{-1}$. Also if $l \geqq 3, V=g / r^{2}$ with $g \in L^{\infty}\left(\boldsymbol{R}^{l}\right)$, then $V$ defines an operator in $L\left(H^{1}, H^{-1}\right) \cdot(-1 / 2(m+i \varepsilon)) \Delta$ may also be regarded as in $L\left(H^{1}, H^{-1}\right)$. Thus the sum of these two operators is in $L\left(H^{1}, H^{-1}\right)$.

In the case $\varepsilon>0$, the method of Theorem 1 applies, since $A=$ $(i / 2(m+i \varepsilon)) \Delta$ is of type $S$ with $S-\{0\}$ contained in the open left half-plane. Thus $(i / 2(m+i \varepsilon)) \Delta-i V \in L\left(H^{1}, H^{-1}\right)$ has a restriction to an operator in $H$ which generates a contraction semigroup.

In the case $\varepsilon=0$, we may use the method of Theorem 2. Here we let $\sigma A=\Delta$ and $\alpha A=(i / 2 m) \Delta$. We need to be able to choose our $H^{1}$ norm in such a way that $V \in L\left(H^{1}, H^{-1}\right)$ has operator norm less than $(1 / 2 m)$. The only additional requirement needed in the examples treated is that $g$ in $L^{\infty}$ have small enough bound. The special case when $l=3$ and $V=-1 / r^{2}$ has been previously treated by Mott and Massey [23, Chap. 2, §6], Case [5], and Nelson [24].

Now we are prepared to see under what circumstances the Trotter formula may be applied to give a Feynman integral representation of the solution.

In the situation studied by Kato, Trotter's results apply directly to give convergence. For example, if $l=3$, a suitable $V$ is a func- 
tion on $\boldsymbol{R}^{3}$ bounded except for finitely many singularities no worse than $1 / r^{s}, s<3 / 2$.

In the more general situation where the Friedrichs extension method is used, we need to ensure that $V$ as an operator in $H$ has domain of definition dense in $H^{1}$.

If $V$ on $\boldsymbol{R}^{l}, l \geqq 3$, is locally in $L^{2}$ except on a discrete set of points $E$, then this condition is satisfied.

Proof. Let $M$ be the space of $C^{\infty}$ functions with compact support in $\boldsymbol{R}^{l}-E . \quad M \subset D(V)$. To show $M$ is dense, let $\nu$ be in $H^{-1}$ with $(\nu, M)=0$.

$\nu$ is a distribution on $\boldsymbol{R}^{l}$. Let $S$ be the support of $\nu$, that is, the smallest closed set $S$ such that $\nu$ is zero on $R^{l}-S$.

$S \subset E$. For consider any $x \notin E$. Since $(\nu, M)=0, \nu$ is zero in some neighborhood of $x$. Thus $x \notin S$.

Since $\nu$ is concentrated on isolated points, $\nu$ is a linear combination of derivatives of $\delta$ measures. But these are in $H^{-1}$ (when $l>1$ ) only if they are zero (as may be seen by taking Fourier transforms.) Thus $\nu=0$.

More generally, we have the following result.

Lemma 3. If $V$ on $\boldsymbol{R}^{l}, l \geqq 3$, is locally in $L^{2}$ except on a compact set $E$ of capacity zero, then $V$ acting in $H$ is defined on a dense subspace of $H^{1}$.

Proof. Let $E$ be a compact set of capacity zero. This means that there is no measure $\mu$ of finite energy supported on $E$, where the energy of $\mu$ is

$$
\frac{1}{2} \iint \frac{1}{|x-y|^{l-2}} d \mu(x) d \mu(y)
$$

In terms of Fourier transforms the energy may be expressed as proportional to

$$
\int|\widehat{u}(x)|^{2} \frac{1}{|x|^{2}} d x
$$

Now a possible norm for $H^{-1}$ might give the distribution $u$ the norm

$$
\int|\widehat{u}(x)|^{2} \frac{1}{|x|^{2}+a^{2}} d x
$$

for some $a>0$. If $u$ happens to be a measure of compact support, the $H^{-1}$ norm of $u$ may be written 


$$
\iint K(x-y) d u(x) d u(y)
$$

where $K(x)$ is the Fourier transform of $\left(1 /|x|^{2}+a^{2}\right)$. This Fourier transform may be evaluated explicitly in terms of Bessel functions (Gel'fand and Shilov [11, p. 288]). Their series expansions show that

$$
K(x)=\frac{1}{|x|^{l-2}} h(|x|),
$$

where $h$ is continuous and $h(0) \neq 0$. Thus if we use the $H^{-1}$ norm to define new notions of energy and capacity, the compact sets of capacity zero are the same.

Thus $E$ is of capacity zero with respect to $K(x)$, that is, there are no measures of finite $H^{-1}$ energy supported on $E$. The new kernel $K(x)$ satisfies the maximum principle (Deny [7, p. 129]). It follows that $E$ must have zero spectral measure (Deny [7, p. 144]). This means that there are no distributions of finite $H^{-1}$ energy supported on $E$.

Now Let $M$ and $\nu$ in $H^{-1}$ be as before. The same proof shows that $\nu$ has support contained in $E$. Thus $\nu=0$.

The following result illustrates the Feynman integral representation of the solution of the Schrödinger equation in a case to which the theory of Kato and Trotter doesn't apply.

THEOREM 3. Let $H=L^{2}\left(\boldsymbol{R}^{l}\right), l \geqq 3$. Let $V$ be a real function which is a finite sum of functions in $L^{p}\left(\boldsymbol{R}^{l}\right), l / 2 \leqq p \leqq \infty$. Assume that $V$ is locally in $L^{2}\left(\boldsymbol{R}^{l}\right)$ except on a compact set of capacity zero. Then for $t \geqq 0$,

$$
\begin{gathered}
\lim _{\varepsilon \rightarrow 0+} \lim _{n \rightarrow \infty} \int \cdots \int \exp \left[i \sum_{j=1}^{n}\left\{\frac{1}{2}(m+i \varepsilon) \frac{\left|x_{j}-x_{j-1}\right|^{2}}{(t / n)^{2}}-V\left(x_{j}\right)\right\} t / n\right] \\
u\left(x_{n}\right)(2 \pi i t / n(m+i \varepsilon))^{-1 / 2 l n} d x_{1} \cdots d x_{n}
\end{gathered}
$$

converges in $H$ to a function $u\left(x_{0}, t\right)$. The correspondence $u(x) \rightarrow u(x, t)$, defines the same one-parameter unitary group of operators as is given by the Friedrichs method.

Proof. Previous results may be used with $A=i \Delta, \sigma=-i, \alpha=$ $(1 / 2(m+i \varepsilon)), B=-i V$. First Theorem 1 and the lemma above apply to $\alpha A=(i / 2(m+i \varepsilon)) \Delta$ (with $\varepsilon>0$ ) and $B$. Thus if $\hat{C}_{n}=\alpha \hat{A}+\hat{B}$ is the sum of their incarnations as operators from $H^{1}$ to $H^{-1}$, we have

$$
\lim _{n \rightarrow \infty}(\exp (t / n \alpha A) \exp (t / n B))^{n}=\exp t C_{\alpha}
$$

in the strong topology. Then Theorem 2 shows that when $\varepsilon \rightarrow 0+$, 
$\exp t C_{\alpha}$ converges strongly to $\exp t C_{(1 / 2 m)}$.

Note that under the same hypotheses we have convergence of

$$
\begin{aligned}
\lim _{n \rightarrow \infty} \int \varphi(m) d m \int & \cdots \int \exp \left[i \sum_{j=1}^{n}\left\{\frac{1}{2} m \frac{\left|x_{j}-x_{j-1}\right|^{2}}{(t / n)^{2}}-V\left(x_{j}\right)\right\} t / n\right] \\
& u\left(x_{n}\right)(2 \pi i t / n m)^{-l n / 2} d x_{1} \cdots d x_{n}
\end{aligned}
$$

where $\phi \in L^{1}$. For the technique, which is to use the Poisson kernel representation of an analytic function of $m$, see Feldman [9, p. 261].

An example of $V$ in the case $l=3$ which is more general than could be treated without this method would be a function bounded on $R^{3}$ except for finitely many singularities no worse than $1 / r^{2-\delta}, \delta>0$. Another example, not included in the theorem as stated, would be $V=g / r^{2}$, where $\|g\|_{\infty}<1 / 4$.

This work was done under the direction of Professor Edward Nelson at Princeton. I want to express my deep gratitude to him for strong encouragement and consistently good advice.

\section{REFERENCES}

1. D. Babbit, A summation procedure for certain Feynman integrals, J. Mathematical Phys. 4 (1963), 36-41.

2. - The Wiener integral and perturbation theory of the Schrödinger operator, Bull. Amer. Matn. Soc. 70 (1964), 254-259.

3. A. V. Balakrishnan, Fractional powers of closed operators and the semigroups generated by them, Pacific J. Math. 10 (1960), 419-437.

4. R. H. Cameron, The Ilstow and Feynman integrals, J. Analyse Math. 10 (1962/ 1963), 287-361.

5. K. M. Case, Singular potentials, Phys. Rev. 80 (1950), 797-806.

6. Yu. L. Daletzski, Continuous integrals associated with operator evolution equations, Uspehi Mat. Nauk 17 (1962), 3-115.

7. J. Deny, Les Potentiels d'energie finite, Acta Math. 82 (1950), 107-183.

8. N. Dunford and J. T. Schwartz, Linear Operators, Part 1: General Theory, Interscience, New York, 1958.

9. J. Feldman, On the Schrödinger and heat equations for non-negative potentials, Trans. Amer. Math. Soc. 108 (1963), 251-264.

10. R. P. Feynman, Space-time approach to non-relativistic quantum mechanics, Rev. Mod. Phys. 20 (1948), 367-387.

11. I. M. Gel'fand and G. E. Shilov, Generalized Functions, Vol. 1, Academic Press, New York, 1964.

12. I. M. Gel'fand and A. M. Yaglom, Integration in function spaces and its application in quantum physics, J. Mathematical Phys. 1 (1960), 48-69.

13. E. Hille and R. S. Phillips, Functional Analysis and Semigroups, Amer. Math. Soc. Colloq. Publ. Vol. 31, Providence, R. I., rev. ed., 1957.

14. K. Ito, Wiener integral and Feynman integral, Proc. Fourth Berkeley Symposium on Math. Statistics and Probability, Vol. II, University of California Press, Berkeley, 1961, 228-238.

15. M. Kac, Probability and Related Topics in the Physical Sciences, Interscience, New York, 1959. 
16. T. Kato, Fractional powers of dissipative operators, J. Math. Soc. Japan 13 (1961), 246-274.

17. Fundamental properties of Hamiltonian operators of Schrödinger type, Trans. Amer. Math. Soc. 70 (1951), 195-211.

18. S. Lang, Introduction to Differentiable Manifolds, Interscience, New York, 1962.

19. J. L. Lions, Equations Differentielles Operationelles, Springer-Verlag, Berlin, 1961. 20. - Espaces d'interpolation et domaines de puissances fractionnaires d'operateurs, J. Math. Soc. Japan 14 (1962), 233-241.

21. G. Lumer and R. S. Phillips, Dissipative operators in a Banach space, Pacific J. Math. 11 (1961), 679-698.

22. E. J. MeShane, Integrals devised for special purposes, Bull. Amer. Math. Soc. 69 (1963), 597-627.

23. N. F. Mott and H. S. Massey, The Theory of Atomic Collisions, 2nd. ed., Clarendon Press, Oxford, 1949.

24. E. Nelson, Feynman Integral and the Schrödinger equation, J. Mathematical Phys. 5 (1964), 332-343.

25. - Operator Differential Equations, Notes for Mathematics 520 (1964-1965), Princeton University, by John Cannon.

26. L. Nirenberg, On elliptic partial differential equations, Annali della Scuola Normale Superieur Pisa 13 (1959), 1-48.

27. H. F. Trotter, Approximation of semi-groups of operators, Pacific J. Math. 8 (1958), 887-919.

28. - On the product of semi-groups of operators, Proc. Amer. Math. Soc. 10 (1959), 545-551.

29. K. Yosida, Functional Analysis, Springer-Verlag, Berlin, 1965.

Received June 28, 1966.

CORNELL UNIVERSITY

ITHACA, NEW YORK 


\section{PACIFIC JOURNAL OF MATHEMATICS}

\section{EDITORS}

\section{H. SAMELSON}

Stanford University

Stanford, California

J. P. JANS

University of Washington

Seattle, Washington 98105
J. Dugundji

University of Southern California Los Angeles, California 90007

RICHARD ARENS

University of California

Los Angeles, California 90024

\section{ASSOCIATE EDITORS}
E. F. BECKENBACH
B. H. NeumanN
F. WOLF
K. YosidA

\section{SUPPORTING INSTITUTIONS}

UNIVERSITY OF BRITISH COLUMBIA

CALIFORNIA INSTITUTE OF TECHNOLOGY

UNIVERSITY OF CALIFORNIA

MONTANA STATE UNIVERSITY

UNIVERSITY OF NEVADA

NEW MEXICO STATE UNIVERSITY

OREGON STATE UNIVERSITY

UNIVERSITY OF OREGON

OSAKA UNIVERSITY

UNIVERSITY OF SOUTHERN CALIFORNIA
STANFORD UNIVERSITY

UNIVERSITY OF TOKYO

UNIVERSITY OF UTAH

WASHINGTON STATE UNIVERSITY

UNIVERSITY OF WASHINGTON

AMERICAN MATHEMATICAL SOCIETY CHEVRON RESEARCH CORPORATION TRW SYSTEMS

NAVAL ORDNANCE TEST STATION 


\section{Pacific Journal of Mathematics \\ Vol. 22, No. $1 \quad$ January, 1967}

Charles A. Akemann, Some mapping properties of the group algebras of a

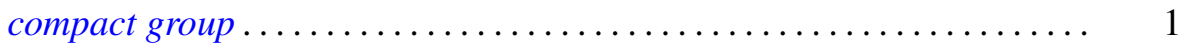

A. V. Boyd, Note on a paper by Uppuluri ..................... 9

Thomas Craig Brown, A semigroup union of disjoint locally finite subsemigroups which is not locally finite .....................

Richard Thomas Bumby and Everett C. Dade, Remark on a problem of

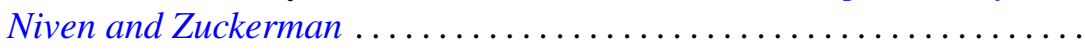

James Calvert, An integral inequality with applications to the Dirichlet problem .........................................

Jack Gary Ceder and Terrance Laverne Pearson, On products of maximally resolvable spaces ....................................

William Guignard Faris, The product formula for semigroups defined by

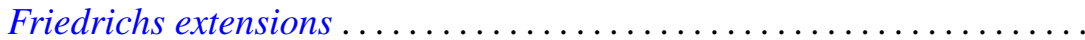

Robert S. Freeman, Closed operators and their adjoints associated with elliptic differential operators ........................ 71

Thomas Lee Hayden, The extension of bilinear functionals ............. 99

Gloria Conyers Hewitt, Limits in certain classes of abstract algebras . . . . . 109

Tilla Weinstein, The dilatation of some standard mappings ........... 117

Mitsuru Nakai, On Evans' kernel ......................... 125

Ernest Levane Roetman, On the biharmonic wave equation ............ 139

Malcolm Jay Sherman, Operators and inner functions ... . .

Walter Laws Smith, On the weak law of large numbers and the generalized elementary renewal theorem

A. J. Ward, On H-equivalence of uniformities: The Isbell-Smith problem 